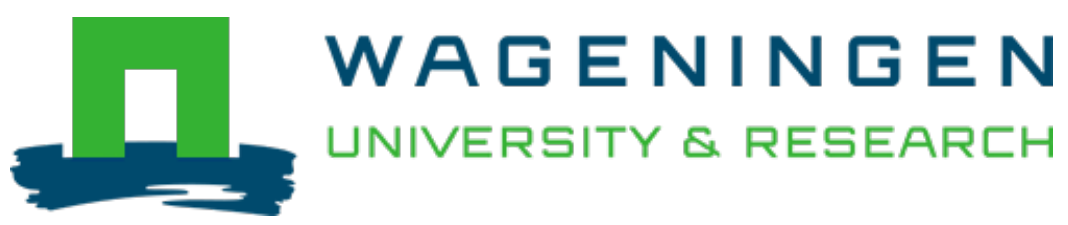

\title{
The contribution of colloidal aggregates to the clogging dynamics at the pore scale
} Journal of Membrane Science

Delouche, N.; Doorn, J.M.; Kodger, T.E.; Schofield, A.B.; Sprakel, J. et al

https://doi.org/10.1016/j.memsci.2021.119509

This article is made publicly available in the institutional repository of Wageningen University and Research, under the terms of article $25 \mathrm{fa}$ of the Dutch Copyright Act, also known as the Amendment Taverne. This has been done with explicit consent by the author.

Article 25 fa states that the author of a short scientific work funded either wholly or partially by Dutch public funds is entitled to make that work publicly available for no consideration following a reasonable period of time after the work was first published, provided that clear reference is made to the source of the first publication of the work.

This publication is distributed under The Association of Universities in the Netherlands (VSNU) 'Article $25 \mathrm{fa}$

implementation' project. In this project research outputs of researchers employed by Dutch Universities that comply with the legal requirements of Article $25 \mathrm{fa}$ of the Dutch Copyright Act are distributed online and free of cost or other barriers in institutional repositories. Research outputs are distributed six months after their first online publication in the original published version and with proper attribution to the source of the original publication.

You are permitted to download and use the publication for personal purposes. All rights remain with the author(s) and / or copyright owner(s) of this work. Any use of the publication or parts of it other than authorised under article $25 \mathrm{fa}$ of the Dutch Copyright act is prohibited. Wageningen University \& Research and the author(s) of this publication shall not be held responsible or liable for any damages resulting from your (re)use of this publication.

For questions regarding the public availability of this article please contact openscience.library@,wur.nl 


\title{
The contribution of colloidal aggregates to the clogging dynamics at the pore scale
}

\author{
N. Delouche ${ }^{\text {a }}$, J.M. van Doorn ${ }^{\text {c }}$, T.E. Kodger $^{c}$, A.B. Schofield ${ }^{\mathrm{b}}$, J. Sprakel ${ }^{\mathrm{c}}$, H. Tabuteau ${ }^{\mathrm{a},}$ \\ ${ }^{a}$ Univ Rennes, CNRS, IPR (Institut de Physique de Rennes)-UMR 6251, F-35000, Rennes, France \\ ${ }^{\mathrm{b}}$ School of Physics and Astronomy, The University of Edinburgh, The James Clerk Maxwell Building, Peter Guthrie Tait Road, Edinburgh, EH9 3FD, UK \\ ${ }^{\mathrm{c}}$ Physical Chemistry and Soft Matter, Wageningen University and Research, Stippeneng 4, 6708, WE Wageningen, the Netherlands
}

\section{A R T I C L E I N F O}

\section{Keywords:}

Colloidal particles

Aggregates

Pore clogging

Microfluidics

Particle size and type distributions

\begin{abstract}
A B S T R A C T
During the filtration of colloidal dispersions by a membrane, pores often get clogged by the suspended particles. Knowing the shape and size of the particles that cause this clog would be a great help to membrane users since they could then choose the ideal filtering device. Microfluidic technology enables the fabrication of model membranes or filters that are transparent, which allows for measuring the particle geometrical features that deposit either at the surface of the pores or on top of the fouling layer that has already formed. However, the use of microfluidic filters have been confined to the study of clog formation at the pore scale, overlooking the influence of the dynamics of the particle deposition on the clogging process. We have recently shown that looking precisely at what is deposited and how this is captured inside the pore provides new insight into the clogging process. In particular, we have found that a minute concentration of aggregates in a supposedly monodisperse dispersions are mainly responsible for pore fouling. In this paper, we use the same imaging technique to determine the entire clogging process for different types of monodisperses dispersions under various flow conditions, DLVO interactions with the pore walls, and confinements. We show that the way clogs form is appear complex but is also quite systematic in the fact that aggregates are the building blocks of the clog. Pores are clogged by progressive accumulation of aggregates with the average size of the aggregate required to cause the blockage increasing with increasing flow velocity. This work demonstrates that particle size and shape distributions of the feeding dispersion must be determined to understand which physical mechanisms are at play during the clogging process.
\end{abstract}

\section{Introduction}

Even though the consequences of membrane fouling on the flow conditions across a membrane are well documented, the precise underlying mechanisms remain poorly understood. We know that deposition of solids carried by a fluid at the surface of a membrane or inside its porous structure leads to a drop of pressure, but we have not yet determined the dynamics of particle deposition or capture by the membrane, up to the complete fouling of the membrane. This difficulty comes from the fact that it is rather challenging to see through a membrane as a suspension flows through it, since membranes are optically opaque. We can still monitor the deposit growth in situ using sophisticated techniques [1-3] but the temporal dynamics of the clogging formation remains inaccessible. We do not have access to the solid particle dynamics in the vicinity of a membrane except in model situations, i.e., with model filters like micro-sieves or microfluidics channels [4-8]. For instance, Valencia et al. were able to determine the dynamics of the very first stages of the cake formation on the top of a membrane with particles bigger than the pore size. Only a few confocal studies have allowed imaging of the entire clogging process at the particle level inside a pore [9-11]. However, these works deal with very narrow pores where only the interaction between the particles and the pore surface are responsible for the fouling, i.e., the pore clogging resulted exclusively from the fouling of the pore surfaces. As far as we are aware, there is no experimental evidence at the pore scale of the clogging process that relies on the progressive accumulation of multilayers of particles either inside a microfluidic model filter or membrane. This type of study is challenging to carry out since three advanced experimental conditions have to be met. Firstly, the particle deposit must be transparent to have access to the internal structure of the

\footnotetext{
* Corresponding author.

E-mail address: herve.tabuteau@univ-rennes1.fr (H. Tabuteau).
} 
particle deposit as it grows unless one uses confocal microscopy with very restrictive conditions on the nature of the suspending liquid and particles [11]. Secondly, accessing the dynamics of the deposit growth requires high speed imaging, around ten thousand frames per second to be able to image the particle capture by the deposit [9]. Finally, measuring the variations of the flow conditions across the pore during fouling is required to determine the hydrodynamic resistance of the deposit. As a starting point to tackle these arduous issues, we have recently focused on the dynamics of the particles capture by the forming deposit [12]. We detect all the particles that go across a single pore. This configuration enables us to follow the trajectory of the particles about to be captured and also their size and shape. In addition, we can determine the probability of capture for the different type of particles, stabilized either by surface charges or polymer brushes.

We have discovered that when a rather monodisperse latex suspension flows through our model filter, the pores are clogged by an accumulation of aggregates and not by single particles, even though aggregates represent only small fraction of the suspension. In this paper, we use this new approach to determine the entire clogging formation for a wide range of flow conditions, using various monodisperse latex suspensions with different surface charges flowing through a model filter. During these experiments, we monitor the deposition history of colloidal particles within the pore and the mean size of each deposited object, which allows us to determine different clogging scenarios. We also determine different features of the deposit stability as it grows. In particular, we monitor detachment events of single particles or aggregates, which helps to quantify the erodibility of the deposits by the fluid flow. We show that for each type of dispersion that pore clogging always results from aggregate accumulation whatever the flow conditions. We distinguish two clogging regimes depending on the flow conditions. At low flow rates, pores are either clogged by an accumulation of small aggregates or by a mixture of small and large aggregates, while at higher flow rates, most of the clogs are formed with large aggregates. The transition between these two regimes is related to the detachment of small aggregates by the flow while the larger ones remain more firmly attached.

\section{Materials and methods}

\subsection{Microfluidics filter and image analysis}

We used the methodology developed in Delouche et al. [12] to make our model microfluidic filter. All pore walls are made of PDMS to obtain the same particle-surface interactions everywhere on the pore surface [11]. During the filtration experiments particles flow through a main wide channel before entering the filter zone composed of long independent pores in parallel (Fig. 1). In this zone, there are two reservoirs upstream of the constriction/pore, which is the thinnest part of the filter and where clogging takes place. The two reservoirs are long and wide enough to ensure that the particle accumulation after the pore clogging event remains located in these reservoirs. Thus in such a configuration we can safely consider that the clogging process for each individual pore is independent of what happens in the surrounding pores. In addition, we can easily track particles over long distances in these two reservoir zones enabling a more accurate determination of their shape. The length of the reservoir also allows us proper tracking of the particles prior to their transport inside the pore. The experiment is stopped when the six pores at the center of the model filter clog. Most of the time not more than half of the pores are clogged when we stop the experiments. We image the transport of the different type of colloidal particles with a sensitive camera (Hamamatsu Orca Flash 4.0 v2). The suspension is injected in the filter by applying a difference of pressure with a regular pressure controller (Elveflow OB1 Mk2 or Fluigent MSFC). We determine the corresponding flow rate at the beginning of the experiment for each applied pressure with a sensor flow (Fluigent, 5\% accuracy) located at the outlet of the filter. Particles follow fluid streamlines and their inertia is negligible since the Péclet and Reynolds numbers for the different flow conditions are respectively higher than $10^{3}$ and smaller than $10^{-3}$. In addition, we use different mixtures of liquid to match the particle density with that of the fluid mixture. We use homemade $1.8 \mu \mathrm{m}$ PMMA and $2.4 \mu \mathrm{m}$ PS particles coated by a polymer brush and commercial suspensions of $1 \mu \mathrm{m}$ and $1.8 \mu \mathrm{m}$ PS particles stabilized by sulfate groups on their surface, bought from Microparticles $\mathrm{GmbH}$ (Berlin). Details on the synthesis of $1.8 \mu \mathrm{m}$ PMMA particles can be found in Delouche et al. [12]. PS coated particles were synthesized using a type of dispersion polymerization where the dispersant is formed in-situ [13]. During the formation of these particles, a reactive initiator-monomer, or inimer, is added which allows for a controlled growth of charged polymer brush by ATRP; the resulting particles have a well-defined charge density. Other $1.8 \mu \mathrm{m}$ and $1 \mu \mathrm{m}$ PS latex particles, stabilized by sulfate groups on their surface, were bought from Microparticles $\mathrm{GmbH}$ (Berlin).

\subsection{Suspensions characterization}

We use the stop and go method to obtain the particle size and shape

a

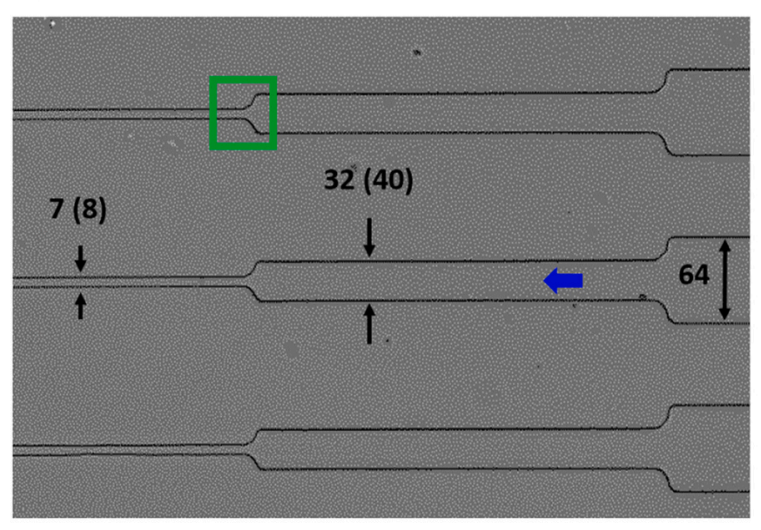

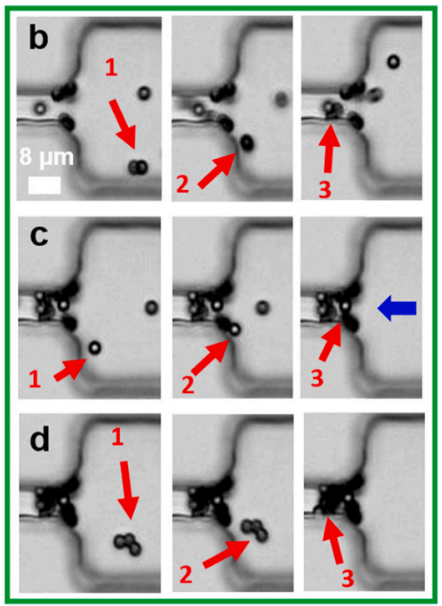

Fig. 1. (Left) A Zoom image of three independent parallel channels in the filter zone. The thinnest part on the left corresponds to the pore/constriction while the two consecutive reservoirs are on the right are where we capture the dynamics of the particles about to deposit in the pore. The numbers in parentheses are related to the larger pore width used with PVP coated PS particles while the other numbers correspond to the pores used in the PMMA and PS particle experiments. All the dimensions are given in $\mu \mathrm{m}$. The blue arrow indicates the flow direction. (Right) Zoom on the constriction/pore zone where the deposition of particles/aggregates takes place with the same flow direction as in (a). Three series of images along the clog formation with 2.4 $\mu \mathrm{m}$ polymer coated PS particles. In the top (b) and middle (c) series a doublet and a single particle, respectively, are captured by one of the pore walls while in the bottom series (d), near the end of the clogging process, a triplet is captured by the deposit. In each series particles were tracked automatically, indicated by the red arrows, up to their deposition. (For interpretation of the references to colour in this figure legend, the reader is referred to the Web version of this article.) 
distribution for all the suspensions we used [12]. Briefly, each suspension is injected inside a straight channel (500 $\mu \mathrm{m}$ wide and $20 \mu \mathrm{m}$ high) and we applied a rectangular wave pressure such that the suspension first does not move for few seconds allowing us to take non-blurred images of the suspended particles. Then the suspension flows for 3-4 $\mathrm{s}$, to renew the particles in the field of view, and the flow is stopped again, and another image of the particles is taken. In this way, we obtain the size and the shape of thousands to millions of particles for each suspension. Such a high particle number counting enables us to easily detect the aggregates present in the suspension thanks to a Fast Radial Symmetry algorithm (FRS) [14]. Whether for the commercial or the synthetized monodisperse suspensions, aggregate particles represent but a few percent of the particle population (Fig. 2, top). Whatever their size, aggregates have very diverse shapes (Fig. 2a-b), and we define only two-dimensional features of each object, $L_{\max }$ and $L_{\min }$ (Fig. 2c). To obtain these lengths, we compute the convex hull of each object which can be simply conceptualized by a rubber band released around the aggregate. $L_{\max }$ also known as the Feret diameter, is the largest antipodal pair of the convex hull, and $L_{\min }$ the smallest $[15,16]$.

We also determine the shape of the particles that get captured inside the pore during the clogging experiments with FRS. These size and shape characterizations are coupled with a particle tracking algorithm in the reservoir before entering the constriction (Fig. 1b-d). Due to the opacity of the deposit, the particle tracking does not directly allow knowledge of what object is deposited. Instead, we subtract the number of each type of deposited object manually from all objects in the reservoir. It is worth noting that we cannot get the precise 3D position of the particle center with this approach since we do not know at which height the particles are deposited, relative to the top or bottom part of the pore.

\section{Results}

\subsection{Influence of particle type and confinement ratio $W / D$ on the overall clogging dynamics}

We have shown recently that minute quantities of aggregates inside a stable and highly monodisperse colloidal suspension deposit more frequently than the spherical particles [12]. In this previous article we focused on the dynamics of the capture of single aggregates directly on the pore walls or at the surface of the colloidal deposit. We also provided some qualitative information that explains why aggregates have such a high probability of capture. Here, we use the same experimental approach to study the dynamics of successive deposition of single particles/aggregates leading to complete pore blocking for different types of particles, various flow conditions and two degrees of confinement defined as the ratio of the pore width, $W$, to the particle size, $D, W / D$. We first used monodisperse dispersions of $1.8 \mu \mathrm{m}$ PMMA and PS particles and $1 \mu \mathrm{m}$ PS particles and flow them in a microfluidic filter with a pore width $W=8 \mu \mathrm{m}$ and $H=12 \mu \mathrm{m}$. We begin by focusing on the global dynamics of pore clogging by looking at the evolution of the average number of particles that flow through the pore prior to clogging, $N^{*}$, with the flow rate $Q$ [17-20] (Fig. 3). We determine $N^{*}$ either by tracking all the particles that flow inside the pore, or from the knowledge of the clogging time and the volume fraction of the suspension [19]. For all the suspensions we used the average value of $N^{*}$ is almost constant for low $Q$ values while it increases for higher ones, as already observed with other particles [11,19]. In this new work, after careful inspection of the dynamics of all the particles that flow through the pore, we observe that there is indeed a progressive clogging of the pore, irrespective of the flow rate. However, most of the particles that form the clog are not single particles but rather aggregate particles, even though all the suspensions we used are primarily composed of individual particles (Fig. 2, top). The growth of the deposit up to complete pore blocking is mainly due to the progressive accumulation of aggregates, between two to six, irrespective of the flow rate and the particle type (Fig. 3, right). It is worth noting that for each suspension and flow condition there are large variations of $N^{*}$ showing that pores are clogged in many different ways, involving aggregates with various shapes and sizes (Fig. 2, bottom).

Hereafter, we determine the different parameters that explain (i) such fluctuations for each $Q$ value and (ii) the evolution of $N^{*}$ with the flow rate. We consider separately regimes of high $(W / D=4.5)$ and low $(W / D=8)$ confinement for the $1.8 \mu \mathrm{m}$ PMMA-PS and $1 \mu \mathrm{m}$ PS particles, respectively. For the higher confinement, we mainly focus on PMMA particles, since similar conclusions can be drawn for PS particles. Despite the high variability in the clog composition, it is still possible to define different categories of clogs, irrespective of the flow conditions, based on geometrical features of the deposited aggregates. We split our clogging experiments into two categories depending on the size of aggregate present within the clog, by considering the greatest dimension of the aggregates, the length $L_{\max }$ that rules out the probability of aggregate capture [12]. Since aggregates have an anisotropic shape they rotate around their center of mass as they flow through our model filter. During this rotation, their two opposite edges along $L_{\max }$ come closer to either

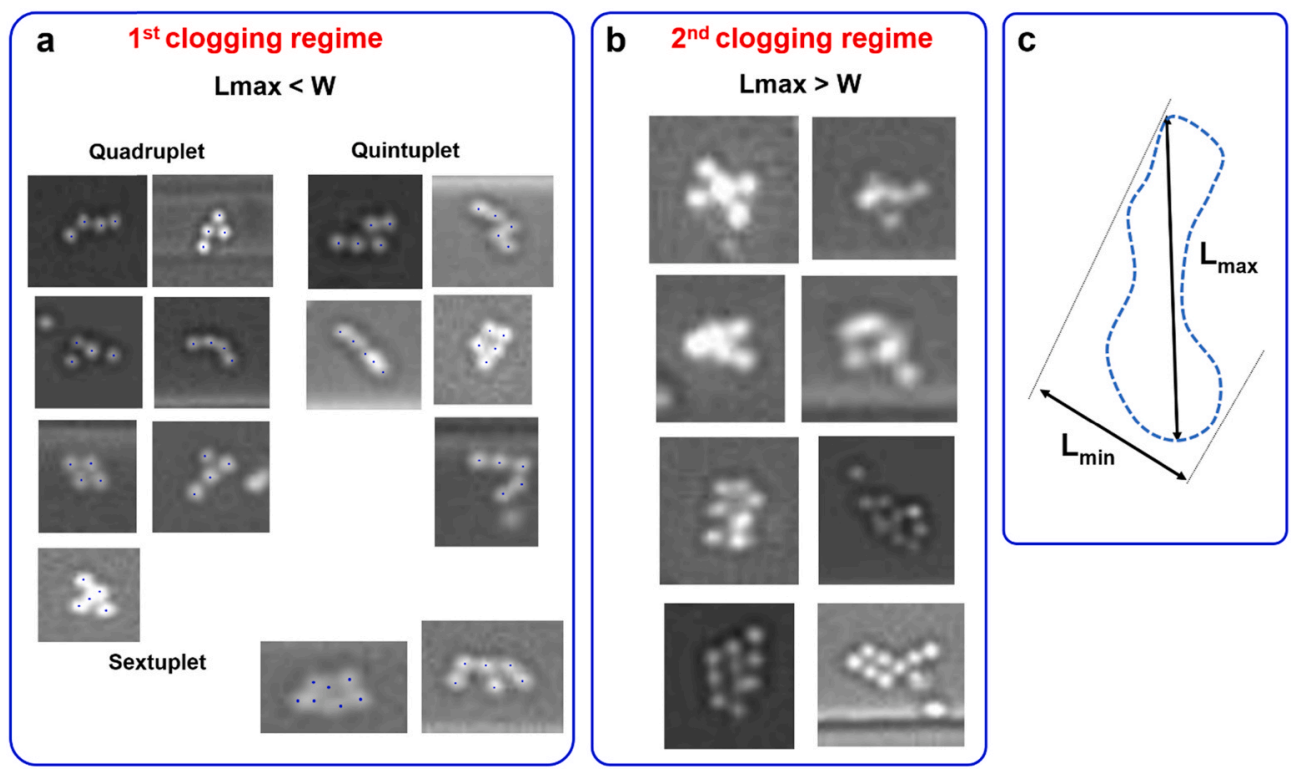

Fig. 2. (Top) Composition of the different suspensions and relative proportion of single particles and aggregates. We work with 6 suspensions of $1.8 \mu \mathrm{m}$ PS particles containing different amount of added $\mathrm{NaCl}$ salt (top) while we work with three suspensions of 2.4 $\mu \mathrm{m}$ PS coated particles exhibiting different surface charges with no addition of salt (bottom right). (Bottom) (a-b) Various fluorescent images of $1.8 \mu \mathrm{m}$ PMMA aggregates in the two clogging regimes with specific conditions of the aggregates length $L_{\max }$. (c) Definition of the length $L_{\max }$ and the width $L_{\min }$ of the aggregates. 

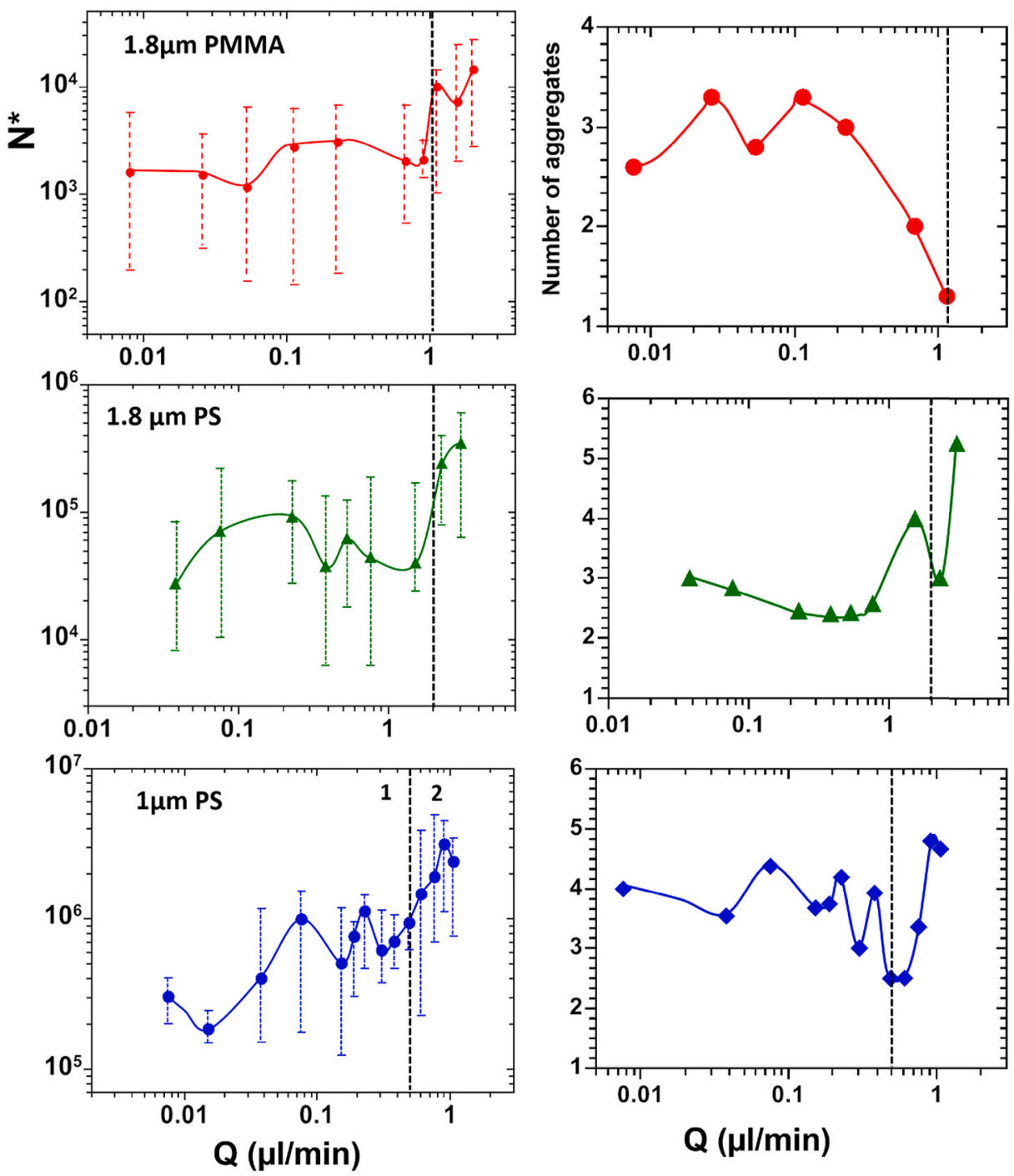

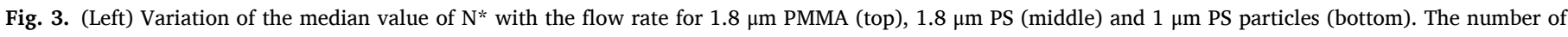

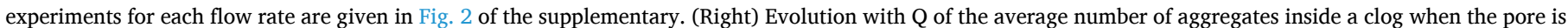

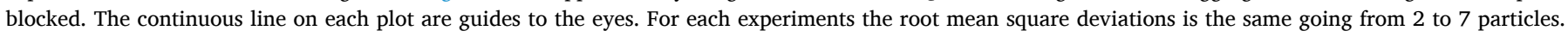

the pore walls or the colloidal deposit and thus increase their probability to touch the surface, which eventually leads to the aggregate capture. We consider that aggregates larger than the pore width, $L_{\max } \geq W$, have the greatest chance to be captured near the pore entrance, by the lateral sides of the pore. In our case this configuration corresponds to $L_{\max } \approx 8$ $\mu \mathrm{m}$. Note that aggregates with a length $L_{\max }=8 \mu \mathrm{m}$ can be composed of 5-7 individual particles, depending on the shape of the aggregates. We found that in the first clog category, every deposited aggregate had its length $L_{\max }<8 \mu \mathrm{m}$. In the second category, there is at least one aggregate with a $L_{\max }>8 \mu \mathrm{m}$. Thus, we divide our results into two distinct regimes of clogging when $L_{\max }<W$ and $L_{\max }>W$.

Figs. 4 and 5 contain consecutive images of the entire clogging process for the 1st and the 2 nd clog categories, respectively. Frequently single particles, the most numerous in the suspensions, start to be captured at the pore walls and are unable to form a thicker deposit (Fig. 4, image 1 to 4 ). It should be noted that aggregates are often captured by the pore walls as well. As discussed elsewhere [11,12,19], the presence of this monolayer of single or aggregate particles greatly enhances the capture of flowing particles. Aggregates, regardless of their dimension, can deposit at any time whatever the degree of fouling (sup. Fig. 1). In addition, not all captured particles belong to the clog, i.e. they are not inside the portion of the deposit that eventually blocks the pore.
Indeed, while most of the particles are captured near the pore entrance over a distance between 20 and $30 \mu \mathrm{m}$ into the pore, the length over which clogs are built corresponds roughly to the pore width, $W$ [19] (last image of Figs. 4 and 5). The final location of the clog and the entire dynamics of the clog formation are not predictable; the growth of the deposit being highly dependent on the local morphology of the deposit, the distance between the flowing aggregates from the deposit surface, and the shape and size of the aggregates [12]. Therefore, even if the deposit becomes important at a given location, reducing appreciably the pore cross section, the final position of the clog can be eventually located elsewhere (Fig. 5).

In the 1st category, $L_{\max }<W$, there is often no more than two large aggregates from quadruplet to sextuplet, which partake in the clog build-up. Even with the deposition of such large aggregates, the pore remains partially clogged and then other smaller aggregates block the remaining pore space, leading to the complete obstruction of the pore (sup. Fig. 1a-b). In the 2nd category, even though the greatest aggregate dimension can be larger than the pore width, $L_{\max }>W$, almost no clogging event is produced by the sieving of a single aggregate. More frequently an aggregate, larger than a quadruplet, is deposited and leads to an important reduction of the pore space, such that only a few objects are needed to terminate the clogging process. Other large aggregates can 


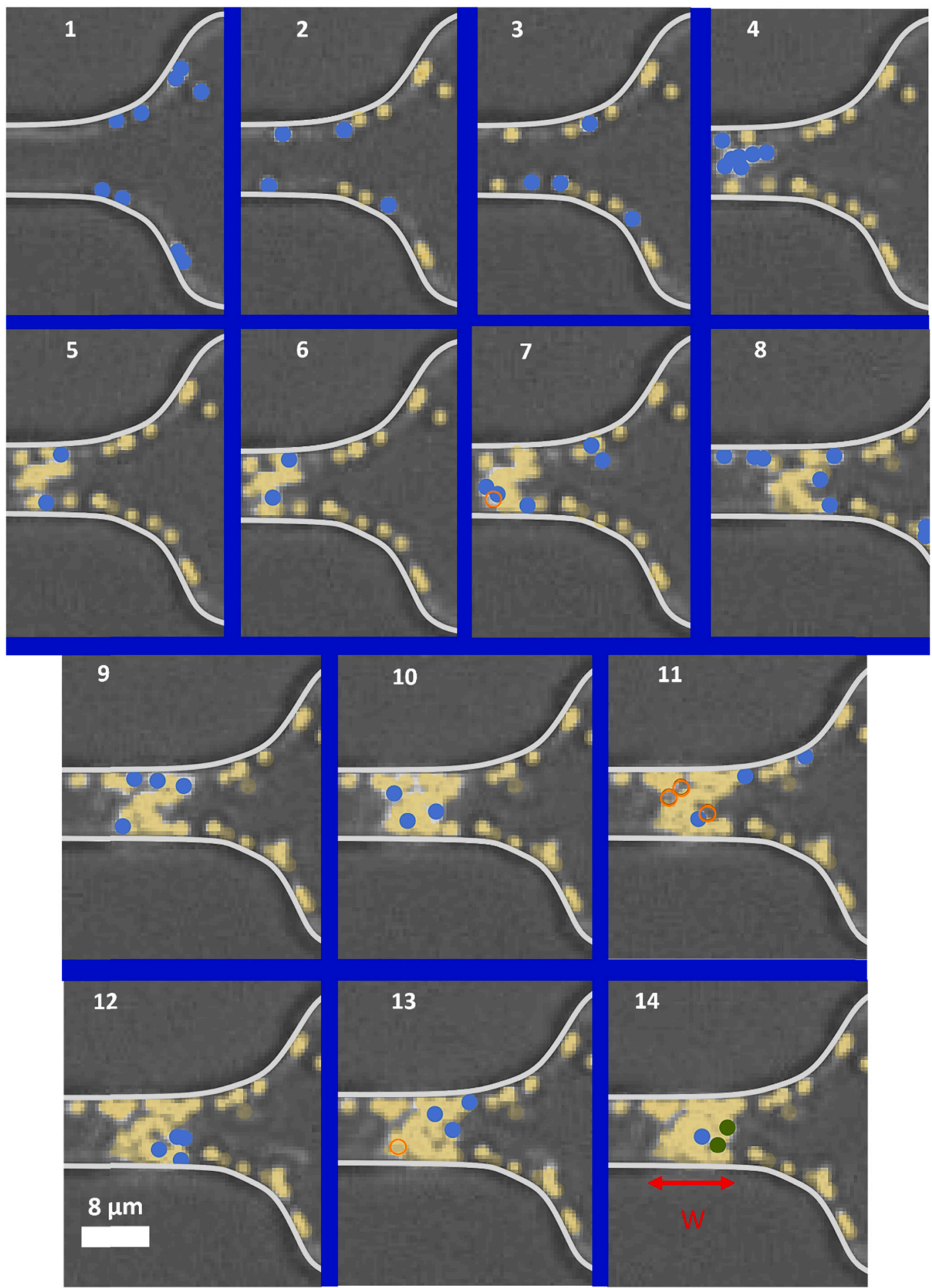

Fig. 4. Consecutive images of the clog formation for the 1st type of clogging corresponding to the data of sup. Fig. 1b. The blue objects correspond to particles that are just being captured while the yellow ones are already deposited. In images 11 and 13, the open circles denote particles detached by the flow. In image 14, the pore is finally blocked by the blue particle, the length of the clog being equal to W. Thereafter the accumulation of particles starts at the rear of the clog (two green particles). (For interpretation of the references to colour in this figure legend, the reader is referred to the Web version of this article.)

also pile up and completely block the pore in this way (sup. Fig. 1c-d).

In the following, we determine the relationship between the occurrences of the two clogging categories and the flow conditions. For the lower flow rates, $0.01<Q<1 \mu \mathrm{l} / \mathrm{min}, N^{*}$ is constant, around 2000 particles, a value high enough to observe several dozens of doublets, a few triplets and larger aggregates that flow through the pore according to the particle size distribution (Fig. 2, top), confirming that the pore can be blocked by aggregate deposition. For these low flow conditions, clogs mainly belong to the first category but as $Q$ increases this trend is inversed (sup Fig. 2, top-left). For $Q>1 \mu \mathrm{l} / \mathrm{min}$, there is an important increase of $N^{*}$ with $Q$ (Fig. 3, top left) and almost all the clogged pores belong to the 2nd category. This indicates that larger aggregates are more involved in the clogging process at higher flow rates. All together these results suggest that the mean size of deposited aggregates increases 


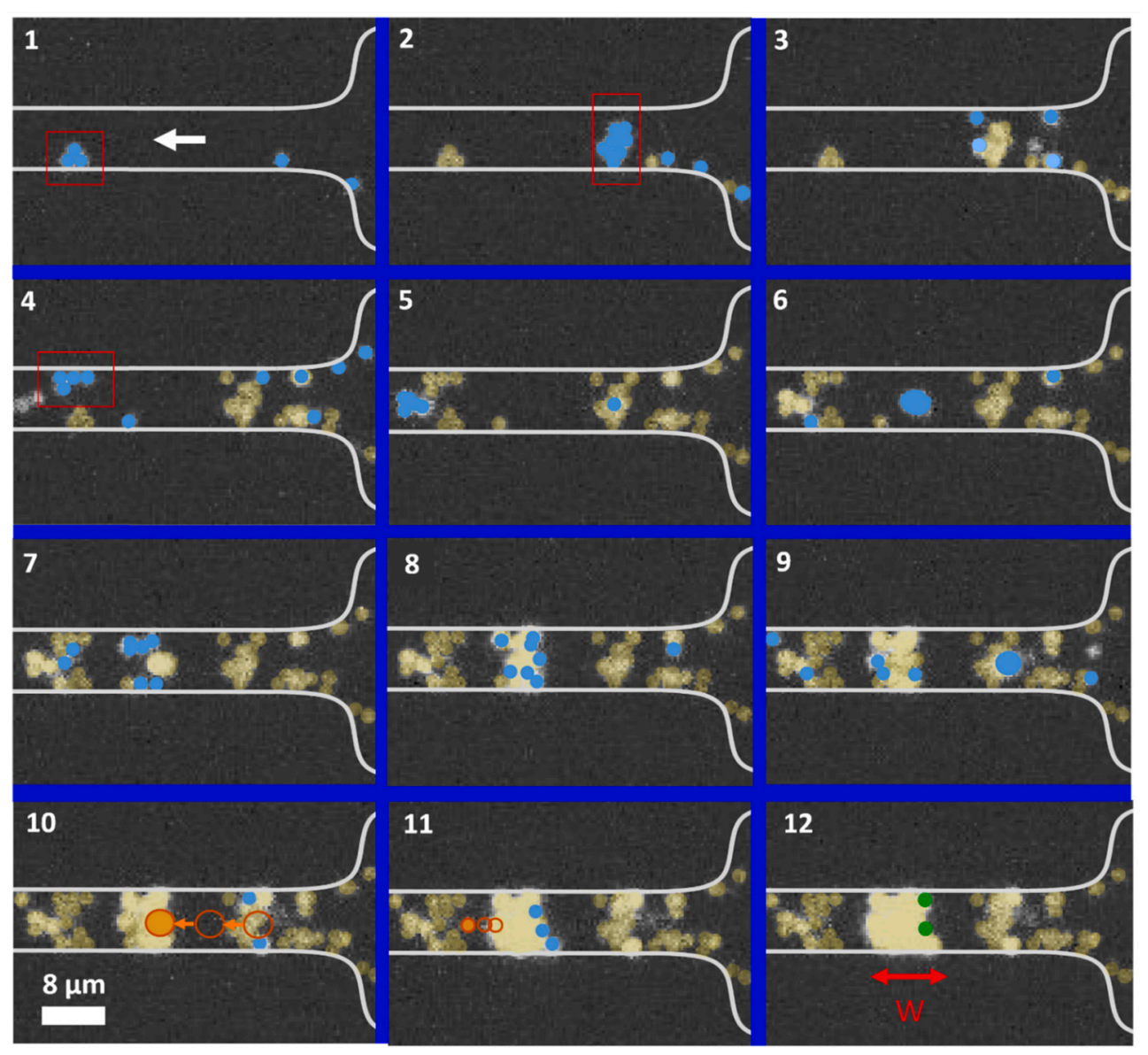

Fig. 5. Consecutive images of the clog formation for the 2nd type of clogging. The blue objects correspond to those particles which are just being captured while the yellow to green ones are already deposited. The rectangles in images 1-2 and 4 highlight the final position of various aggregates whose deposition dynamics can be found in Delouche et al. [12]. In image 9, a large particle is captured and remains static for $2 \mathrm{~s}$ before it again moves towards the location where the pore will eventually clog (image 10). In image 11, there are rearrangements within the clog that lead to the expulsion of particles at the clog front which do not seem to weaken the internal structure of the clog. In image 12, the pore is completely blocked; the length of the clog being equal to $\mathrm{W}$ and then the accumulation of particles starts at the rear of the clog (two green particles). (For interpretation of the references to colour in this figure legend, the reader is referred to the Web version of this article.)

with $Q$, evolution that can be understood if we consider the erosion by the fluid flow. At high flow rate the erosion prevents mainly the deposition of single particles and small aggregates, which thus leads to an increase of $N^{*}$ since the clogging process mainly relies on large aggregates that are less numerous and thus requires that more particles flow through the pore prior to aggregate capture. A more detailed discussion in provided in the supplementary material (sup Fig. 3).

When we consider the PS particles, with the same size as the PMMA particles, we observe the same trend for the evolution of $N^{*}$ with $Q$. However, the average value of $N^{*}$ in the 1 st clogging regime is 2.5 times higher than for PMMA particle, which can be explained mainly by the difference in distribution of particle size between the two suspensions. Indeed, for this suspension the total amount of aggregate number is only $0.56 \%$ while for the PMMA this is equal to $3.17 \%$ (Fig. 2, top) and hence more PS particles must flow through the pore to observe aggregate deposition. There is also an important variation of the particle size/ object distribution. The proportion of large aggregates, which includes quadruplets, is equal to $0.37 \%$ for the PMMA while it is much smaller for PS, around to $0.01 \%$. However, among this population of large aggregates for PS particles there are few very larges objects that alone can plug the pore. These particles are responsible for $37 \%$ of the total clogging events at low flow rates, which increases significantly the number of clogs belonging to the 2nd clog category compared to the PMMA case (sup Fig. 2, top right). The increases of $\mathrm{N}^{*}$ with the flow rate starts from a higher $Q$ value, presumably because PS particles have an increased adhesion on the pore walls and also with each other [19], and thus higher flow conditions are needed for particle detachment by the flow to occur.

The situation for the smaller $1 \mu \mathrm{m}$ PS particles is similar despite the degree of confinement being seemingly less favorable to the formation of clogs since more particles being required to clog a pore. The average number of aggregates to block the pore is slightly larger than for the two other types of particles, being around four (Fig. 3, bottom). Therefore, since the aggregates are formed with smaller particles there are more particles per aggregates, around 12-14 on average. Regardless of flow rate, all the clogs belong to the second category, which means that large aggregates are always involved in the clogging process (sup Fig. 2, bottom). The evolution of the average number of aggregates is similar to that of observed for the $1.8 \mu \mathrm{m}$ PS (Fig. 3, bottom right). Since the particle size distribution is quite monodisperse (Fig. 2), the probability that such large aggregates flow through the pore is low, which explains why the average value of $\mathrm{N}^{*}$ at low flow rate is high, close to $7 \cdot 10^{5}$. (Fig. 3, bottom left).

\subsection{Influence of the ionic strength}

The clogging dynamics are modified by changing either the ionic strength of the suspension or the surface charge of the particles. In a first set of experiments involving $1.8 \mu \mathrm{m}$ PS particles, the ionic strength of the suspension was modified by adding $\mathrm{NaCl}$ salt. In such a case, both particles and pore wall surface charges are modified simultaneously. In a second set of experiments, we worked with rather monodisperse polystyrene particles (PS) coated with a PVP brush which imparts steric stabilizations. In these experiments, another polymer is grown from the particle surface but with varying ratio of neutral, cationic and an anionic monomer to create particles of varying surface charge density [13].

\subsection{Change of ionic strength of the suspensions by salt addition}

We used the classical strategy to screen the surface charge the $1.8 \mu \mathrm{m}$ PS particles, simply by adding $\mathrm{NaCl}$ salt to the particle suspension. Before each experiment the model filter is saturated by a salty solution 
with the same salt concentration, $C$, as that of the colloidal suspension about to be injected. For $C$ above $50 \mathrm{mM}$ and $200 \mathrm{mM}$, respectively, the surface charge of the pore walls and that of the particles is completely screened. The particle size and type distribution in the various salty suspensions were determined thanks to the stop and go experiments previously described (Fig. 2, top). For $C \leq 100 \mathrm{mM}$ the population of aggregates are slightly modified, while above $100 \mathrm{mM}$ it rises up significantly, with a steeper increase of the number of aggregates larger than quadruplets (Fig. 6a-b).

In the clogging experiments the salt addition has a direct impact on the evolution of $N^{*}$ with flow rate (Fig. 6c-d). For the first clogging regime, $Q<1 \mu \mathrm{l} / \mathrm{min}, N^{*}$ decreases with $C$, with the sharpest decrease, found when the salt concentration comes closer to the critical coagulation concentration (CCC) of the suspension, $C=200 \mathrm{mM}$ (Fig. 6d). While $N^{*}$ does not vary with the flow conditions for the $C=0 \mathrm{mM}$ dispersion, it first increases and then becomes constant for increasing $Q$ for all the salty dispersion. These variations are far less pronounced for $C=200$ $\mathrm{mM}$. These evolutions of $N^{*}$ are again directly related to the relative proportion of the aggregate population inside the suspensions (Fig. 2, top). The average number of aggregates required to clog a pore remains constant as long as the concentration of aggregates slightly increases, for $C$ smaller than $100 \mathrm{mM}$ (sup Fig. 4c). Even though the size of the aggregates involved in the clog formation significantly increases for $C>$ $100 \mathrm{mM}$, the clogs are formed with more aggregates. The measure of the relative proportion of the two clogging categories defined previously indicates that the average size of the aggregates forming the clogs increases with both the flow rate and the salt concentration (see sup. Fig. 4 for a detailed description).

\subsection{Influence of the surface charge of the particles}

We performed clogging experiments with suspensions of $2.4 \mu \mathrm{m}$ PS particles with an anionic, cationic and neutral surface charges polymer coating for which the confinement ratio is higher than previously, $W / D$ $=3.3$ and with no salt addition. In such a high confinement the clog formation depends chiefly on the interactions between the surface of the particles and the pore walls rather than on the interparticle interactions [11]. In a given cross section only two monolayers need to deposit on both pore walls before only one extra layer of particle in between them is needed to block the pore almost completely. Thus, we may expect that the pores will be clogged more rapidly by the cationic particles since there is a net attraction between these particles and the negatively charged pore surface. Neutral particles should also rapidly clog the pores since they do not bear surface charges. We indeed observe that deposition of single particles directly on the pore surface is two times higher for the neutral and cationic particles than for the anionic ones. However, when we look at the overall clogging dynamics, the surface charge of the particles does not appear to play a role since the average number of particles needed to block the pore, $N^{*}$, is about the same, around 2000 particles for each particle type (Fig. 7a).

Looking at the particle level and the type of particles, we found again that aggregates play a dominant role in the clogging process. Since the confinement is higher, with $W / D=3.3$, there is more particle capture by the pore walls and the average number of aggregates that deposit inside the pore, is also twice as high compared to other particles tested in section 2 of this paper (Fig. 7c). This can also come, in a lesser extent, from the higher aggregate concentration in the coated PS particles, around $7-8 \%$ of the total number of particles (Fig. 2, top). For the anionic particles, there are on average more than four large aggregates (quadruplets or larger) within each clog (Fig. 7c) which represent around $6 \%$ of the total amount of deposited particles within the clogs while they are the least numerous population of the suspension, less than $0.4 \%$ (Fig. 2, top). This is due to their high probability of capture (Fig. 7b). The situation is a bit different for the two other types of
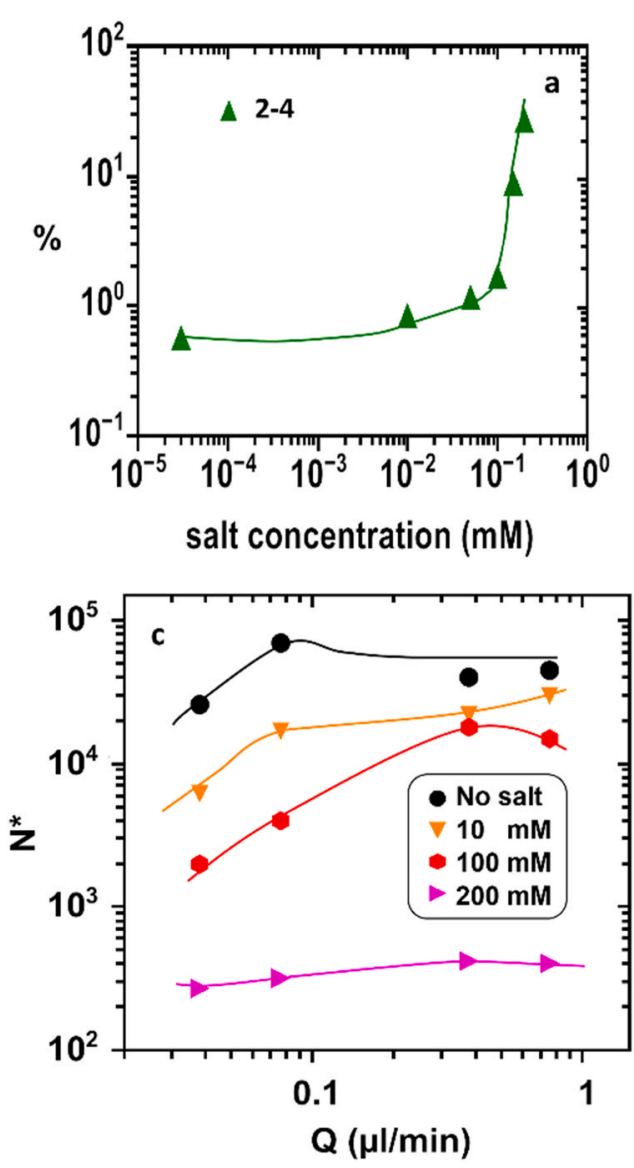

Fig. 6. Particle population percentage of doublet up to quadruplet (a) and larger aggregates bigger than quadruplets (b) measured during the stop and go experiments for suspensions of $1.8 \mu \mathrm{m}$ PS with various amount of salt. (c) Variation of $\mathrm{N}^{*}$ with $\mathrm{Q}$ for suspensions with ionic strength. The data for the suspension with no salt added is identical to those shown in Fig. 3 (middle, left). (d) Evolution of N* averaged for all $\mathrm{Q}$ values vs. the salt concentration. The continuous lines on all plots are guides to the eyes. (For interpretation of the references to colour in this figure legend, the reader is referred to the Web version of this article.) 

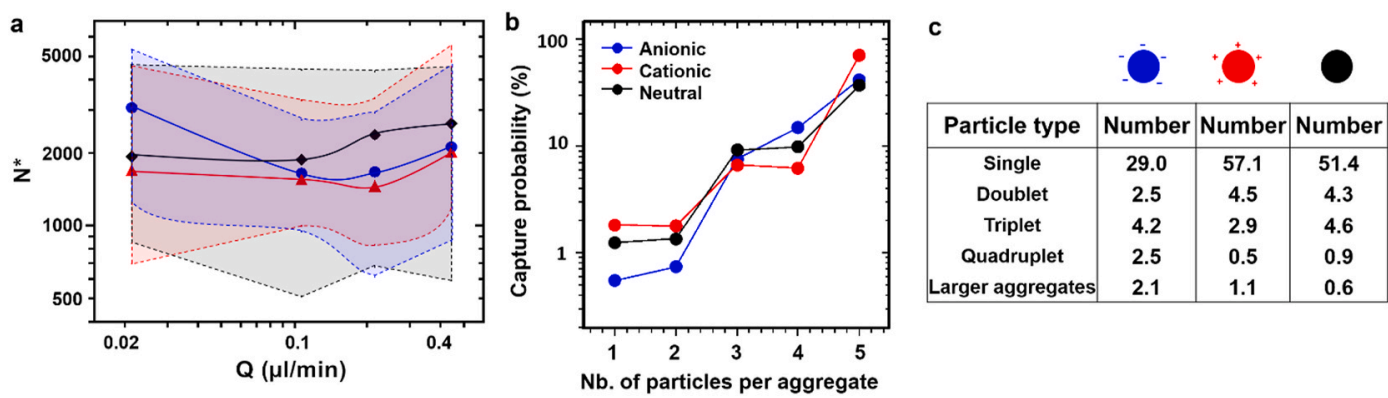

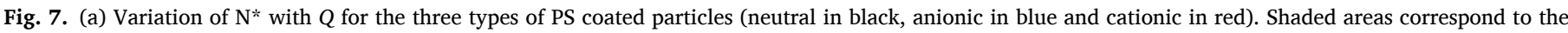

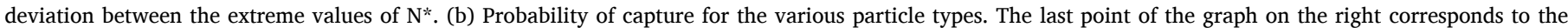

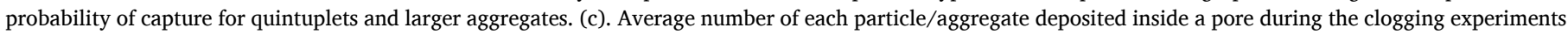
for all the flow rates. (For interpretation of the references to colour in this figure legend, the reader is referred to the Web version of this article.)

particles. For cationic and neutral particles there are only 1.5 aggregates larger than triplets in each clog on average (Fig. 7c). Note that significant part of the pores is clogged only by the accumulation of single particles and small aggregates, doublets and triplets. This is directly related to both the relative proportion of single particles and doublet in the suspensions and the surface charge properties of these suspensions. The population of single particles and doublets corresponds to $98.4 \%$ of the total amount of particles for both suspensions (Fig. 2, top) which increases their probability of capture. In addition, there is no electrostatic barrier between the pore surface and the neutral particles and even a net attraction for the cationic ones since the pore walls are negatively charged [21] promoting the particle deposition of both types of particles, since their capture probability is roughly 5 times higher than for the anionic particles (Fig. 7b). We supposed that neutral particles are captured only by physical interception, while the anionic and cationic particles are driven towards the pore surfaces due to very short-range electrostatic attractions, $30-50 \mathrm{~nm}$ from the pore surface. Therefore, cationic particles that are further away from the pore walls than the neutral ones can still deposit, which explains why the capture probability is slightly higher for cationic particles (Fig. 7b).

Surprisingly, these favorable conditions for particle deposition for the cationic and neutral particles do not play a role when we consider larger aggregates, starting from triplets. Indeed, the probability of capture for these aggregates are roughly the same, irrespective of the surface charge (Fig. 7b). This capture probability does not depend on the aggregate shape since aggregates have on average the same shape for the different particle types (sup Fig. 5). We suppose that the motion of large aggregates inside the pore is responsible of their capture. These aggregates are captured by physical interception as they rotate, when one of their edges come into contact with either the pore wall or the deposit [12]. Our results suggest that the nature of the surface charge does not modify the capture dynamics of large aggregates, since the capture probability is the same whatever the particle type. Therefore, the presence of large aggregates inside clogs formed by anionic particles is only due to the higher quantity of such aggregates in the anionic suspension injected in the model filter.

\section{Discussion}

In this work we considered fouling within the pore rather than on top of the membrane since the size of the particles of our suspensions are smaller than the pore size. We identified the different clogging mechanisms when the feeding suspensions contains aggregates and particles smaller and/or larger than the mean pore size of the filter. Pores can first be blocked by sieving a large particle or an aggregate. However, there are still some free spaces between the pore surface and this big object in which small particles can flow through and eventually block them. A second mechanism relies on successive accumulation of smaller aggregates, which is the more common case in our experiments. Whatever the mechanism, even though single spherical particles can also be involved in the clogging process, they cannot alone block the pore by successive accumulation. They are mainly captured directly on the pore walls, by physical interception, or by the deposit, likely by sieving. Indeed, we have not observed physical interception of single particles by those which belong to the monolayer in contact with the pore walls or at the top of a deposit, while such a mechanism of particle capture is commonly used in numerical simulations of pore clogging [22,23]. However, from our experiments we cannot conclude definitively that there is no physical interception of a flowing particle by another immobile one, but rather that physical interception of aggregates is far more likely than physical interception of single particles. In the light of these results, it may be useful to have a new look at the results of almost all the works related to the clogging dynamics and mechanisms that used microfluidic channels as a model filter and monodisperse suspensions of spherical colloids $[17,19,20,24-28]$ as the filtrate. Indeed, the results of these studies rely on the implicit hypothesis that single particles accumulate one after the other forming the deposit and thus they have not considered that aggregates could have played any role in the fouling process. In particular, we think that clogging of small pore sizes, not larger than two particle diameters, is more likely the result of doublet or larger aggregates capture rather than the hydrodynamic bridging of two single particles that enter simultaneously inside the pore [26,28-31]. Indeed, irrespective of the chemical synthesis of monodisperse particles, there are always small aggregates like doublets or even triplets in the suspensions. For instance in all the suspensions we used, the population of doublets is far from negligible since doublets represent between $0.5 \%$ and $8 \%$ of all the particles. This means that from a dozen up to 200 particles of these dispersions pass through a filter with pore sizes around two particle diameters doublets may statistically be present and can block the pores at their entrance. Therefore, even if there is only a tenth of a percent of aggregates in a suspension the probability to block the pore by aggregate sieving should be far more important than clogging the pore by hydrodynamic bridging. New experimental investigations involving the imaging of the particle capture inside or at the pore entrance have to be performed to confirm the sieving of aggregates in very confined situations.

\section{Conclusion}

By observing the dynamics of particle deposition within a pore, we show that for different types of monodisperse colloidal suspensions, under various flow conditions, confinements and electrostatic interactions, clogging proceeds mainly by successive depositions of aggregated particles. Therefore, even though these aggregates represent only few percent of the population they represent a major impact on the clogging process. We considered the clogging within the pore rather than on top of the membrane since the size of the particles of our feeding suspensions are smaller than the pore size. We identified two main 
mechanisms of pore clogging. Pores can first be blocked by sieving a large particle or an aggregate. However, there are still some free spaces between the pore surface and this big object in which small particles can flow through and eventually block them. A second mechanism relies on successive accumulation of smaller aggregates, which is the more common case in our experiments.

We show that when an average number of particles $\mathrm{N}^{*}$, or equivalently a Critical Volume of Suspension (CVS), passes through a pore it becomes clogged. Contrary to other works in dead end configurations $[26,27]$ we found that there is always a CVS above which the pore will be completely fouled, irrespective of the flow conditions. We show that the CVS is directly related to the concentration of aggregates in the suspension. The CVS is constant for lower pressures while it increases with the pressure for higher pressures. Since we performed on average around fourteen replicates for each pressure, we can observe that for the range of pressure we used pores can be clogged either by accumulation of aggregates or by a partial fouling of the pore followed by the sieving of a large aggregate. The proportion of each category strongly depends on the aggregate size distribution of the different suspensions, however the average size of the aggregates involved in the clog formation always increases with increasing pressure. This trend is due to the detachment by flow of the smaller aggregates since they have less contact points with the deposit.

The role of aggregates in the clogging process has also to be confirmed in other pore geometries, especially for those encountered in industrial filters and membranes. Here the maximum average speed of the particles is not greater than $7 \mathrm{~mm} / \mathrm{s}$, a value which is comparable to those measured inside microfiltration membranes with the same pore size, around $10 \mathrm{~mm} / \mathrm{s}$ [28]. Therefore, we expect that such membranes will be clogged mainly by the sieving of large aggregates rather than by the successive deposition of small aggregates which are smaller than the mean pore size.

Since our model filters are deformable, we cannot perform backwashing experiments to determine the mechanical resistance of the different types of clog. However, our results suggest that even if most of the particles forming the clog can be removed aggregates lying on the pore surface will rarely be detached by the flow. Finally, we believe that pore clogging by accumulation of aggregates should also be considered in cross flow conditions.

\section{Author statement}

N. Delouche: Conceptualization, Methodology, Investigation, Writing - Original Draft, Visualization, Writing - Review \& Editing, Formal analysis. J.M. van Doorn, T.E. Kodger, A.B. Schofield and J. Sprakel: Methodology, Writing - Review \& Editing, Formal analysis. H. Tabuteau: Conceptualization, Methodology, Investigation, Writing Original Draft, Visualization, Writing - Review \& Editing, Formal analysis. Supervision, Project administration, Funding acquisition.

\section{Declaration of competing interest}

The authors declare that they have no known competing financial interests or personal relationships that could have appeared to influence the work reported in this paper.

\section{Acknowledgments}

We acknowledge funding from the French Agence Nationale de la Recherche (ANR) (ANR-12-JS09-0003) and the CNES (Collmat).

\section{Appendix A. Supplementary data}

Supplementary data to this article can be found online at https://doi. org/10.1016/j.memsci.2021.119509.

\section{References}

[1] Y. Gao, S. Haavisto, W. Li, C.Y. Tang, J. Salmela, A.G. Fane, Novel approach to characterizing the growth of a fouling layer during membrane filtration via optical coherence tomography, Environ. Sci. Technol. 48 (2014) 14273-14281, https:// doi.org/10.1021/es503326y.

[2] W. Luo, B. Arhatari, S.R. Gray, M. Xie, Seeing is believing: insights from synchrotron infrared mapping for membrane fouling in osmotic membrane bioreactors, Water Res. 137 (2018) 355-361, https://doi.org/10.1016/j. watres.2018.03.011.

[3] I. Ben Hassan, C. Lafforgue, A. Ayadi, P. Schmitz, In situ 3D characterization of monodispersed spherical particle deposition on microsieve using confocal laser scanning microscopy, J. Membr. Sci. 454 (2014) 283-297, https://doi.org/ 10.1016/j.memsci.2013.12.003.

[4] I.S. Ngene, R.G.H. Lammertink, M. Wessling, W. van der Meer, A microfluidic membrane chip for in situ fouling characterization, J. Membr. Sci. 346 (2010) 202-207, https://doi.org/10.1016/j.memsci.2009.09.035.

[5] I.S. Ngene, R.G.H. Lammertink, M. Wessling, W.G.J. Van der Meer, Visual characterization of fouling with bidisperse solution, J. Membr. Sci. 368 (2011) 110-115, https://doi.org/10.1016/j.memsci.2010.11.026.

[6] A. Valencia, C. Le Men, C. Ellero, C. Lafforgue-Baldas, P. Schmitz, J.F. Morris, Direct observation at the microscale of particle deposition during the first stage of the microfiltration process, J. Membr. Sci. 599 (2020) 117823, https://doi.org/ 10.1016/j.memsci.2020.117823.

[7] J. Lin, D. Bourrier, M. Dilhan, P. Duru, Particle deposition onto a microsieve, Phys. Fluids 21 (1994-Present) (2009), 073301.

[8] G. Brans, A. van Dinther, B. Odum, C.G.P.H. Schroën, R.M. Boom, Transmission and fractionation of micro-sized particle suspensions, J. Membr. Sci. 290 (2007) 230-240, https://doi.org/10.1016/j.memsci.2006.12.045.

[9] B. Dersoir, A.B. Schofield, H. Tabuteau, Clogging transition induced by selffiltration in a slit pore, Soft Matter 13 (2017) 2054-2066, https://doi.org/ 10.1039/C6SM02605B.

[10] J. Linkhorst, J. Rabe, L.T. Hirschwald, A.J.C. Kuehne, M. Wessling, Direct observation of deformation in microgel filtration, Sci. Rep. 9 (2019) 18998 https://doi.org/10.1038/s41598-019-55516-w.

[11] B. Dersoir, A.B. Schofield, M. Robert de Saint Vincent, H. Tabuteau, Dynamics of pore fouling by colloidal particles at the particle level, J. Membr. Sci. 573 (2019) 411-424, https://doi.org/10.1016/j.memsci.2018.12.025.

[12] N. Delouche, A.B. Schofield, H. Tabuteau, Dynamics of progressive pore clogging by colloidal aggregates, Soft Matter 16 (2020) 9899-9907, https://doi.org/ 10.1039/D0SM01403F.

[13] T.E. Kodger, R.E. Guerra, J. Sprakel, Precise colloids with tunable interactions for confocal microscopy, Sci. Rep. 5 (2015) 14635.

[14] G. Loy, A. Zelinsky, Fast radial symmetry for detecting points of interest, IEEE Trans. Pattern Anal. Mach. Intell. 25 (2003) 959-973, https://doi.org/10.1109/ TPAMI.2003.1217601.

[15] F.P. Preparata, M.I. Shamos, Computational Geometry: an Introduction, Springer Science \& Business Media, 2012

[16] IM. Yaglom, VG. Boltyanskii, Convex Figures (Library of Mathematical Circle Volume 4), n.d. https://www.biblio.com/book/convex-figures-librarymathematical-circle-volume/d/1034841221 (accessed December 19, 2020).

[17] H.M. Wyss, D.L. Blair, J.F. Morris, H.A. Stone, D.A. Weitz, Mechanism for clogging of microchannels, Phys. Rev. 74 (2006), 061402.

[18] A. Sauret, E.C. Barney, A. Perro, E. Villermaux, H.A. Stone, E. Dressaire, Clogging by sieving in microchannels: application to the detection of contaminants in colloidal suspensions, Appl. Phys. Lett. 105 (2014), 074101.

[19] B. Dersoir, M.R. de S. Vincent, M. Abkarian, H. Tabuteau, Clogging of a single pore by colloidal particles, Microfluid. Nanofluidics 19 (2015) 953-961, https://doi. org/10.1007/s10404-015-1624-y.

[20] S.S. Massenburg, E. Amstad, D.A. Weitz, Clogging in parallelized tapered microfluidic channels, Microfluid. Nanofluidics 20 (2016) 94, https://doi.org/ 10.1007/s10404-016-1758-6.

[21] E. Spruijt, H.E. Bakker, T.E. Kodger, J. Sprakel, M.A.C. Stuart, J. van der Gucht Reversible assembly of oppositely charged hairy colloids in water, Soft Matter 7 (2011) 8281-8290, https://doi.org/10.1039/C1SM05881A.

[22] G.C. Agbangla, E. Climent, P. Bacchin, Numerical investigation of channel blockage by flowing microparticles, Comput. Fluids 94 (2014) 69-83.

[23] J. Lohaus, Y.M. Perez, M. Wessling, What are the microscopic events of colloidal membrane fouling? J. Membr. Sci. 553 (2018) 90-98, https://doi.org/10.1016/j. memsci.2018.02.023.

[24] T. van de Laar, S. ten Klooster, K. Schroën, J. Sprakel, Transition-state theory predicts clogging at the microscale, Sci. Rep. 6 (2016) 28450, https://doi.org/ 10.1038 /srep28450.

[25] R. van Zwieten, T. van de Laar, J. Sprakel, K. Schroën, From cooperative to uncorrelated clogging in cross-flow microfluidic membranes, Sci. Rep. 8 (2018) 5687, https://doi.org/10.1038/s41598-018-24088-6.

[26] P. Bacchin, A. Marty, P. Duru, M. Meireles, P. Aimar, Colloidal surface interactions and membrane fouling: investigations at pore scale, Adv. Colloid Interface Sci. 164 (2011) 2-11.

[27] G.C. Agbangla, É. Climent, P. Bacchin, Experimental investigation of pore clogging by microparticles: evidence for a critical flux density of particle yielding arches and deposits, Separ. Purif. Technol. 101 (2012) 42-48.

[28] Z.B. Sendekie, P. Bacchin, Colloidal jamming dynamics in microchannel bottlenecks, Langmuir 32 (2016) 1478-1488. 
[29] V. Ramachandran, H.S. Fogler, Multilayer deposition of stable colloidal particles during flow within cylindrical pores, Langmuir 14 (1998) 4435-4444.

[30] V. Ramachandran, H.S. Fogler, Plugging by hydrodynamic bridging during flow of stable colloidal particles within cylindrical pores, J. Fluid Mech. 385 (1999) 129-156, https://doi.org/10.1017/S0022112098004121.
[31] G. Constant Agbangla, P. Bacchin, E. Climent, Collective dynamics of flowing colloids during pore clogging, Soft Matter 10 (2014) 6303-6315, https://doi.org/ 10.1039/C4SM00869C. 